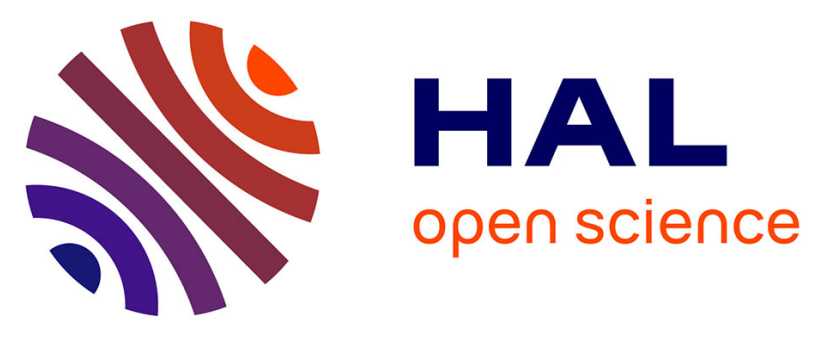

\title{
The ASTRES Toolbox for Mode Extraction of Non-Stationary Multicomponent Signals
}

\author{
Dominique Fourer, Jinane Harmouche, Jérémy Schmitt, Thomas Oberlin, \\ Sylvain Meignen, François Auger, Patrick Flandrin
}

\section{- To cite this version:}

Dominique Fourer, Jinane Harmouche, Jérémy Schmitt, Thomas Oberlin, Sylvain Meignen, et al.. The ASTRES Toolbox for Mode Extraction of Non-Stationary Multicomponent Signals. EUSIPCO 2017 - 25th European Signal Processing Conference, Aug 2017, Kos, Greece. pp.1130-1134, 10.23919/EUSIPCO.2017.8081384 . hal-01579903

\section{HAL Id: hal-01579903 https://hal.science/hal-01579903}

Submitted on 31 Aug 2017

HAL is a multi-disciplinary open access archive for the deposit and dissemination of scientific research documents, whether they are published or not. The documents may come from teaching and research institutions in France or abroad, or from public or private research centers.
L'archive ouverte pluridisciplinaire HAL, est destinée au dépôt et à la diffusion de documents scientifiques de niveau recherche, publiés ou non, émanant des établissements d'enseignement et de recherche français ou étrangers, des laboratoires publics ou privés. 


\title{
The ASTRES Toolbox for Mode Extraction of Non-Stationary Multicomponent Signals
}

\author{
Dominique Fourer ${ }^{\star}$, Jinane Harmouche, Jérémy Schmitt, Thomas Oberlin, \\ Sylvain Meignen, François Auger and Patrick Flandrin \\ *dominique@fourer.fr
}

\begin{abstract}
In this paper, we introduce the ASTRES* toolbox which offers a set of Matlab functions for non-stationary multicomponent signal processing. The main purposes of this proposal is to offer efficient tools for analysis, synthesis and transformation of any signal made of physically meaningful components (e.g. sinusoid, trend or noise). The proposed techniques contain some recent and new contributions, which are now unified and theoretically strengthened. They can provide efficient time-frequency or time-scale representations and they allow elementary components extraction. Usage and description of each method are then detailed and numerically illustrated.
\end{abstract}

\section{INTRODUCTION}

Real world signals are often non-stationary and made of several components. They require advanced techniques to be efficiently processed. Unfortunately, the Short-Time Fourier Transform (STFT) and the Continuous Wavelet Transform (CWT) which belong among the most usual approaches, are limited due to a poor energy localization in the time-frequency plane [1].

Two solutions, the reassignment and the synchrosqueezing methods [2], [3], [4], can improve the readability of a time-frequency representation (TFR). More particularly, the synchrosqueezing can provide sharpened and invertible TFRs allowing many applications like noise removal and signal decomposition into modes [3], [5], [6]. In addition, the Empirical Mode Decomposition (EMD) [7] and the Singular Spectrum Analysis (SSA) [8], which belong to data-driven methods, allow unsupervised signal decomposition into a sum of physically meaningful components (e.g. periodic functions, trend or noise). Several recent developments were proposed to enhance these techniques [9] and to enable a fully automatic version of SSA, in the same flavor as EMD.

This paper proposes a collection of Matlab functions related to the new methods developed in the "Analysis, Synthesis, Transformation by Reassignment, EMD and Synchrosqueezing" (ASTRES) research project*. This new toolbox (cf. Fig. 1), designed for automatic signal mode extraction, can be viewed as an extension of the previously proposed Time-Frequency ToolBox (TFTB $)^{1}$. It contains implementations of some recent contributions related to reassignment, synchrosqueezing [10], [11], [6], [12], EMD [13], [14], and SSA [9] methods. This paper is organized as follows. In Section II, proper definitions

* This research was supported by the French ANR ASTRES project (ANR13-BS03-0002-01).

${ }^{1} \mathrm{http}$ //tftb.nongnu.org, ${ }^{2} \mathrm{https}$ //github.com/dfourer/ASTRES_toolbox

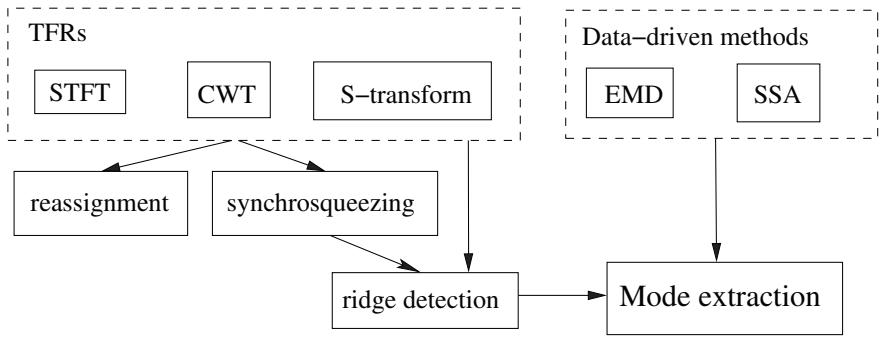

Fig. 1. ASTRES toolbox content description freely available online ${ }^{2}$.

of the considered transforms and their TFRs are presented. In Section III, reassigned and synchrosqueezed versions of each transform are described with two ridge detection methods. Section IV describes data-driven methods, EMD and SSA, with their corresponding new developments. Finally, the proposed methods are illustrated by numerical experiments on real world signal in Section V, before concluding the paper in Section VI. II. TIME-FREQUENCY REPRESENTATIONS

\section{A. Short-time Fourier transform (STFT)}

Let $F_{x}^{h}(t, \omega)$ denote the STFT of signal $x$, using a differentiable analysis window $h$, defined as

$$
F_{x}^{h}(t, \omega)=\int_{\mathbb{R}} x(u) h(t-u)^{*} \mathbf{e}^{-j \omega u} \mathrm{~d} u,
$$

$z^{*}$ being the complex conjugate of $z$. Thus, the spectrogram can be computed by $\left|F_{x}^{h}(t, \omega)\right|^{2}$ (cf. tfrstft, tfrgab). Eq. (1) admits the following synthesis formula when $h\left(t_{0}\right) \neq 0$, allowing to recover signal $x$ with a delay $t_{0} \geq 0$ (cf. rectfrgab)

$$
x\left(t-t_{0}\right)=\frac{1}{h\left(t_{0}\right)} \int_{-\infty}^{+\infty} F_{x}^{h}(t, \omega) \mathbf{e}^{j \omega\left(t-t_{0}\right)} \frac{\mathrm{d} \omega}{2 \pi} .
$$

An efficient recursive implementation (suitable for real-time computation), is proposed in [10], [12]. It uses a specific causal (one-sided) analysis window which can be expressed as $h_{k}(t)=\frac{t^{k-1}}{T^{k}(k-1) !} \mathbf{e}^{-t / T} U(t)$, with $k \geq 1$, where $U(t)$ is the Heaviside step function and $T$ a time spread parameter ( $c f$. recursive_stft and stft_rec).

\section{B. Continuous wavelet transform (CWT)}

The CWT of a signal $x$ is defined for an admissible mother wavelet function $\Psi$ as [1]

$$
W_{x}^{\Psi}(t, s)=\frac{1}{\sqrt{|s|}} \int_{-\infty}^{+\infty} x(\tau) \Psi\left(\frac{\tau-t}{s}\right)^{*} \mathrm{~d} \tau
$$


If we define the scale as $s=\frac{\omega_{0}}{\omega}, \omega_{0}$ being an arbitrary frequency, Eq. (3) can now be expressed as a time-frequency transform as

$$
\mathrm{CW}_{x}(t, \omega)=\sqrt{\frac{|\omega|}{\omega_{0}}} \int_{-\infty}^{+\infty} x(\tau) \Psi\left(\frac{\omega}{\omega_{0}}(\tau-t)\right)^{*} \mathrm{~d} \tau .
$$

The scalogram can be computed by $\left|W_{x}(t, s)\right|^{2}$ or $\left|\mathrm{CW}_{x}(t, \omega)\right|^{2}$. If we use the Morlet wavelet [1], expressed as $\Psi(t)=\frac{\pi^{-1 / 4}}{\sqrt{T}} \mathbf{e}^{\frac{-t^{2}}{2 T^{2}}} \mathbf{e}^{j \omega_{0} t}$, we finally obtain $\operatorname{MW}_{x}(t, \omega)=$

$$
\sqrt{\frac{|\omega|}{\omega_{0} T \sqrt{\pi}}} \int_{-\infty}^{+\infty} x(\tau) \mathbf{e}^{-\frac{\omega^{2}(t-\tau)^{2}}{2\left(\omega_{0} T\right)^{2}}} \mathbf{e}^{-j \omega(\tau-t)} \mathrm{d} \tau
$$

(cf. tfrscalo and MW). The synthesis formula of Eq. (3) is given by ( $c f$. recMW)

$$
x(t)=\frac{1}{C_{\psi}} \int_{\mathbb{R}} W_{x}^{\Psi}(t, s)|s|^{-3 / 2} d s, \text { with } C_{\psi}=\int_{\mathbb{R}} F_{\Psi}(\omega)^{*} \frac{\mathrm{d} \omega}{\omega}
$$

where $F_{\Psi}(\omega)$ denotes the Fourier transform of $\Psi(t)$.

\section{Stockwell transform (S-transform)}

The S-transform [15] can be defined for a given zero-mean signal $x$ as (cf. tfrst)

$$
\operatorname{ST}_{x}(t, \omega)=\frac{|\omega|}{\sqrt{2 \pi} \omega_{0} T} \int_{-\infty}^{+\infty} x(\tau) \mathbf{e}^{-\frac{\omega^{2}(t-\tau)^{2}}{2\left(\omega_{0} T\right)^{2}}} \mathbf{e}^{-j \omega \tau} \mathrm{d} \tau
$$

where $T$ is the width of the Gaussian analysis window when $\omega=\omega_{0}$. The corresponding TFR, called Stockwellogram, is provided by $\left|\mathrm{ST}_{x}(t, \omega)\right|^{2}$. The S-transform can be viewed as a STFT using a frequency-varying window width,

$$
\operatorname{ST}_{x}^{h}(t, \omega)=\frac{|\omega|}{\omega_{0}} \int_{-\infty}^{+\infty} x(\tau) h\left(\frac{\omega}{\omega_{0}}(t-\tau)\right) \mathbf{e}^{-j \omega \tau} \mathrm{d} \tau
$$

where $h$ is a Gaussian window defined as $h(t)=\frac{1}{\sqrt{2 \pi} T} \mathbf{e}^{-\frac{t^{2}}{2 T^{2}}}$. The S-transform can also be related to the Morlet wavelet transform by

$$
\operatorname{ST}_{x}(t, \omega)=\sqrt{\frac{|\omega|}{2 \sqrt{\pi} \omega_{0} T}} \mathbf{e}^{-j \omega t} \operatorname{MW}_{x}(t, \omega),
$$

which shows that the S-transform can be viewed as a normalized phase-shifted Morlet wavelet transform. Thus, substituting $\mathrm{MW}_{x}$ in Eq. (6) leads to the S-transform synthesis formula (cf. rectfrst2) [16]

$$
x(t)=\frac{1}{C_{h}\left(\omega_{0} T\right)} \int_{\mathbb{R}} \operatorname{ST}_{x}(t, \omega) \mathbf{e}^{j \omega t} \frac{\mathrm{d} \omega}{|\omega|},
$$

where $C_{h}\left(\omega_{0} T\right)$ is a proportionality factor defined by

$$
C_{h}\left(\omega_{0} T\right)=\int_{\mathbb{R}} F_{h}\left(\xi-\omega_{0}\right) \frac{\mathrm{d} \xi}{|\xi|} .
$$

\section{REASSIGNMENT AND SYNCHROSQUEEZING}

Reassignment and synchrosqueezing are sharpening techniques designed to improve TFRs. The main difference between these methods is the reconstruction capability of synchrosqueezing and a quite poorer time-frequency localization compared to the reassignment, which is not invertible.

\section{A. Reassignment}

For each transform, the TFR values are moved according to the map $(t, \omega) \mapsto\left(\hat{t}_{(t, \omega)}, \hat{\omega}_{(t, \omega)}\right)$, where $\hat{t}_{(t, \omega)}=\operatorname{Re}\left(\tilde{t}_{(t, \omega)}\right)$ and $\hat{\omega}_{(t, \omega)}=\operatorname{Im}\left(\tilde{\omega}_{(t, \omega)}\right)$ (resp. $\left.\hat{s}_{(t, \omega)}=\operatorname{Im}\left(\tilde{s}_{(t, \omega)}\right)\right)$, are the reassignment operators.

1) STFT: Reassignment operators can be computed as [2]

$$
\tilde{t}(t, \omega)=t-\frac{F_{x}^{\mathcal{T} h}(t, \omega)}{F_{x}^{h}(t, \omega)}, \tilde{\omega}(t, \omega)=j \omega+\frac{F_{x}^{\mathcal{D} h}(t, \omega)}{F_{x}^{h}(t, \omega)},
$$

with $\mathcal{T} h(t)=t h(t)$ and $\mathcal{D} h(t)=\frac{d h}{d t}(t)$. Thus, the reassigned spectrogram can be computed as (cf. tfrrsp and recursive_rsp) $\mathrm{RF}_{x}(t, \omega)=$

$$
\iint_{\mathbb{R}^{2}}\left|F_{x}^{h}(\tau, \Omega)\right|^{2} \delta(t-\hat{t}(\tau, \Omega)) \delta(\omega-\hat{\omega}(\tau, \Omega)) \mathrm{d} \tau \mathrm{d} \Omega .
$$

A recent extension of the reassignment process, based on the Levenberg-Marquardt algorithm, allows to adjust the energy localization in the time-frequency plane through a damping parameter $\mu$ [17]. The new reassignment operators can be computed as

$$
\left(\begin{array}{c}
\hat{t}_{\mu}(t, \omega) \\
\hat{\omega}_{\mu}(t, \omega)
\end{array}\right)=\left(\begin{array}{c}
t \\
\omega
\end{array}\right)-\left(\nabla^{t} R_{x}^{h}(t, \omega)+\mu I_{2}\right)^{-1} R_{x}^{h}(t, \omega)
$$

with $R_{x}^{h}(t, \omega)=\left(\begin{array}{c}t-\hat{t}(t, \omega) \\ \omega-\hat{\omega}(t, \omega)\end{array}\right), \nabla^{t} R_{x}^{h}(t, \omega)=\left(\begin{array}{ll}\frac{\partial R_{x}^{h}}{\partial t}(t, \omega) & \frac{\partial R_{x}^{h}}{\partial \omega}(t, \omega)\end{array}\right)$

where $I_{2}$ is the $2 \times 2$ identity matrix. As a result, the Levenberg-Marquardt reassigned spectrogram can be obtained by replacing $(\hat{t}, \hat{\omega})$ by $\left(\hat{t}_{\mu}, \hat{\omega}_{\mu}\right)$ in Eq. (13) (cf. tfrlmrgab and recursive_lmrsp).

2) CWT: Since, $\frac{\partial}{\partial t} W_{x}^{\Psi}(t, s)=-\frac{1}{s} W_{x}^{\mathcal{D} \Psi}(t, s)$, the CWT reassignment operators can be expressed by [2], [1], [18]

$$
\begin{array}{r}
\tilde{t}(t, s)=t+s \frac{W_{x}^{\mathcal{T} \Psi}(t, s)}{W_{x}^{\Psi}(t, s)}, \\
\tilde{s}(t, s)=\frac{\omega_{0}}{\tilde{\omega}(t, s)}=-\frac{s \omega_{0} W_{x}^{\Psi}(t, s)}{W_{x}^{\mathcal{D} \Psi}(t, s)} .
\end{array}
$$

Then, the reassigned scalogram can be computed as ( $c f . r \mathrm{MW})$ $\mathrm{RW}_{x}(t, s)=$

$$
\iint_{\mathbb{R}^{2}}\left|W_{x}^{\Psi}\left(\tau, s^{\prime}\right)\right|^{2} \delta\left(t-\hat{t}\left(\tau, s^{\prime}\right)\right) \delta\left(s-\hat{s}\left(\tau, s^{\prime}\right)\right) \frac{s^{\prime 2}}{\hat{s}\left(\tau, s^{\prime}\right)^{2}} \mathrm{~d} \tau \mathrm{d} s^{\prime} .
$$

3) S-transform: Reassignment operators can be computed as for the STFT ( $c f$. proofs in $\S 8,[16])$. Thus, both classical and Levenberg-Marquardt reassigned Stockwellograms are obtained through Eqs. (12) and (13), by replacing $F_{x}^{h}(t, \omega)$ by $\mathrm{ST}_{x}^{h}(t, \omega), F_{x}^{\mathcal{T} h}$ by $\mathrm{ST}_{x}^{\mathcal{T} h}$ and $F_{x}^{\mathcal{D} h}$ by $\mathrm{ST}_{x}^{\mathcal{D} h}$ (cf. tfrrst.m and tfrlmrst). Thus we have $\operatorname{RST}_{x}(t, \omega)=$

$$
\iint_{\mathbb{R}^{2}}\left|\mathrm{ST}_{x}(\tau, \Omega)\right|^{2} \delta(t-\hat{t}(\tau, \Omega)) \delta(\omega-\hat{\omega}(\tau, \Omega)) d \tau d \Omega
$$

\section{B. Synchrosqueezing}

Each synchrosqueezed transform can be deduced from the simplified synthesis formula given by Eqs (2), (6) and (10), respectively for the STFT, the CWT and the S-transform. 
1) STFT: The synchrosqueezed STFT can be defined for any $t_{0} \geq 0$ such that $h\left(t_{0}\right) \neq 0$ (cf. tfrsgab, recursive_sstft)

$$
\mathrm{SF}_{x}^{h}(t, \omega)=\int_{\mathbb{R}} F_{x}^{h}\left(t, \omega^{\prime}\right) \mathbf{e}^{j \omega^{\prime}\left(t-t_{0}\right)} \delta\left(\omega-\hat{\omega}\left(t, \omega^{\prime}\right)\right) \mathrm{d} \omega^{\prime},
$$

Its squared modulus provides a sharpened TFR, and it can be inverted by

$$
\hat{x}\left(t-t_{0}\right)=\frac{1}{h\left(t_{0}\right)^{*}} \int_{\mathbb{R}} \mathrm{SF}_{x}^{h}(t, \omega) \frac{\mathrm{d} \omega}{2 \pi},
$$

Replacing $\hat{\omega}$ by $\hat{\omega}_{\mu}$ computed by Eq. (14) allows to make synchrosqueezing adjustable as for the Levenberg-Marquardt reassignment ( $c f$. tfrlmsgab, recursive_lmsstft).

2) $C W T$ : The synchrosqueezed CWT can be defined as [3] (cf. sMW)

$$
\mathrm{SW}_{x}(t, s)=\int_{\mathbb{R}} W_{x}\left(t, s^{\prime}\right)\left|s^{\prime}\right|^{-3 / 2} \delta\left(s-\hat{s}\left(t, s^{\prime}\right)\right) \mathrm{d} s^{\prime},
$$

and can be inverted by ( $c f$. recsMW)

$$
\hat{x}(t)=\frac{1}{C_{\Psi}} \int_{\mathbb{R}} \mathrm{SW}_{x}(t, s) \mathrm{d} s,
$$

3) S-transform: The synchrosqueezed S-transform can be defined as [16] (cf. tfrsst, tfrlmsst)

$\operatorname{SST}_{x}(t, \omega)=|\omega| \int_{\mathbb{R}} \operatorname{ST}_{x}\left(t, \omega^{\prime}\right) \mathbf{e}^{j \omega^{\prime} t} \delta\left(\omega-\hat{\omega}\left(t, \omega^{\prime}\right)\right) \frac{\mathrm{d} \omega^{\prime}}{\left|\omega^{\prime}\right|}$,

and can be inverted by (cf. rectfrsst)

$$
\hat{x}(t)=\frac{1}{C_{h}\left(\omega_{0} T\right)} \int_{\mathbb{R}} \operatorname{SST}_{x}(t, \omega) \frac{\mathrm{d} \omega}{|\omega|},
$$

\section{Second-order synchrosqueezing}

Second-order synchrosqueezing was first proposed by Oberlin et al. in [11] to improve TFRs of strongly modulated chirps signal. Assuming a signal model expressed as

$$
x(t)=a(t) \mathbf{e}^{j \Phi(t)}, \text { with } \Phi(t)=\varphi_{x}+\omega_{x} t+\alpha_{x} \frac{t^{2}}{2},
$$

where $a(t)$ and $\Phi(t)$ stand for the time-varying amplitude and phase. Second-order synchrosqueezing proposes to use local modulation estimation to improve the resulting TFR. Oblique synchrosqueezing (only proposed for the STFT) ( $c f$. tfrosgab) can be viewed as a time-frequency reassignment with a phase correction term [11],

$$
\begin{aligned}
\operatorname{OSF}_{x}(t, \omega)=\iint_{\mathbb{R}^{2}} F_{x}^{h}\left(\tau, \omega^{\prime}\right) \mathbf{e}^{j\left(\omega-\frac{\hat{\alpha}_{x}}{2}(t-\tau)\right)(t-\tau)} & \\
& \delta\left(t-\hat{t}\left(\tau, \omega^{\prime}\right)\right) \delta\left(\omega-\hat{\omega}\left(\tau, \omega^{\prime}\right)\right) \mathrm{d} \tau \mathrm{d} \omega^{\prime} .
\end{aligned}
$$

Vertical synchrosqueezing uses an improved estimation of the instantaneous frequency (instead of the classical reassignment operator), thanks to the chirp rate estimation $\hat{\alpha}_{x(t, \omega)}=$ $\operatorname{Im}\left(\tilde{q}_{x^{(t, \omega)}}\right)$ (see [12] for a further investigation). The improved frequency estimator can be computed as

$$
\hat{\omega}^{(2)}(t, \omega)= \begin{cases}\hat{\omega}_{(t, \omega)}+\hat{\alpha}_{x(t, \omega)}\left(t-\hat{t}_{(t, \omega)}\right) & \text { if }\left|\hat{\alpha}_{x(t, \omega)}\right|<\infty \\ \hat{\omega}(t, \omega) & \text { otherwise }\end{cases}
$$

For amplitude modulated signals, it is shown in [12] that (27) is biased, and can be improved using a slightly different estimator expressed as (when $\left|\tilde{q}_{x(t, \omega)}\right|<\infty$ )

$$
\hat{\omega}^{(3)}(t, \omega)=\operatorname{Im}\left(\tilde{\omega}_{(t, \omega)}+\tilde{q}_{x^{(t, \omega)}}\left(t-\tilde{t}_{(t, \omega)}\right)\right) .
$$

An estimation of $\tilde{q}_{x}(t, \omega)$ can be computed for each transform as follows:

1) STFT: In [12], we proposed several local modulation estimators, valid for any differentiable analysis window, $\forall n \geq$ 2, which can be used in Eq. (27) or in (28) to obtain a vertical synchrosqueezed STFT transform ( $c f$. tfrvsgab,

$$
\begin{aligned}
& \hat{\alpha}_{x}^{K n}(t, \omega)=\frac{\operatorname{Re}\left(F_{x}^{\mathcal{D}^{n} h}(t, \omega) F_{x}^{\mathcal{D}^{n-1} h}(t, \omega)^{*}\right)}{\operatorname{Im}\left(F_{x}^{\mathcal{T} \mathcal{D}^{n-1} h}(t, \omega) F_{x}^{\mathcal{D}^{n-1} h}(t, \omega)^{*}\right)}, \\
& \tilde{q}_{x}^{(t n)}(t, \omega)=\frac{F_{x}^{\mathcal{D}^{n} h}(t, \omega) F_{x}^{h}(t, \omega)-F_{x}^{\mathcal{D}^{n-1} h}(t, \omega) F_{x}^{\mathcal{D} h}(t, \omega)}{F_{x}^{\mathcal{D}^{n-1} h}(t, \omega) F_{x}^{\mathcal{T} h}(t, \omega)-F_{x}^{\mathcal{T} \mathcal{D}^{n-1} h}(t, \omega) F_{x}^{h}(t, \omega)},
\end{aligned}
$$

$$
\tilde{q}_{x}^{(\omega n)}(t, \omega)=\frac{\left(F_{x}^{\mathcal{T}^{n-1} \mathcal{D} h}+(n-1) F_{x}^{\mathcal{T}^{n-2} h}\right) F_{x}^{h}-F_{x}^{\mathcal{T}^{n-1} h} F_{x}^{\mathcal{D} h}}{F_{x}^{\mathcal{T}^{n-1} h} F_{x}^{\mathcal{T} h}-F_{x}^{\mathcal{T}^{n} h} F_{x}^{h}},
$$

where $(t, \omega)$ was omitted in Eq. (31) for the sake of clarity. In [12], these estimators were implemented and compared in terms of accuracy for $n=2$.

2) $C W T$ : Using the local modulation estimator $\tilde{q}_{x}(t, s)=$ $\frac{\frac{\partial \tilde{\omega}}{\partial t}(t, s)}{\frac{\partial t}{\partial t}(t, s)}$ proposed for the CWT [18], we obtain (cf. vsMW)

$$
\tilde{q}_{x}(t, s)=\frac{\frac{1}{s^{2}}\left(W_{x}^{\mathcal{D}^{2} \Psi}(t, s) W_{x}^{\Psi}(t, s)-W_{x}^{\mathcal{D} \Psi}(t, s)^{2}\right)}{W_{x}^{\mathcal{D} \Psi}(t, s) W_{x}^{\mathcal{T} \Psi}(t, s)-W_{x}^{\mathcal{T} \mathcal{D} \Psi}(t, s) W_{x}^{\Psi}(t, s)} .
$$

3) S-transform: A new local modulation estimator can also be derived to compute the vertical synchrosqueezed Stransform [16] (cf. tfrvsst),

$$
\tilde{q}_{x}(t, \omega)=\frac{\mathrm{ST}_{x}^{\mathcal{D}^{2} h}(t, \omega) \mathrm{ST}_{x}(t, \omega)-\mathrm{ST}_{x}^{\mathcal{D} h}(t, \omega)}{\mathrm{ST}_{x}^{\mathcal{D} h}(t, \omega) \mathbf{S T}_{x}^{\mathcal{T} h}(t, \omega)-\mathrm{ST}_{x}^{\mathcal{T} \mathcal{D} h}(t, \omega) \mathbf{S T}_{x}(t, \omega)} .
$$

\section{Ridge detection for mode extraction}

Let us consider a multicomponent signal denoted by $x(t)=$ $\sum_{i=1}^{I} x_{i}(t)$. Then, each mode $x_{i}(t)=a_{i}(t) \mathbf{e}^{j \phi_{i}(t)}$ can be extracted from its synchrosqueezed transform, by restricting the integration area to the vicinity of the ridge, denoted $\Omega_{i}(t)$, in each reconstruction formula given by Eqs. (20), (22) and (24). The ridge $\Omega_{i}(t)$ can be directly estimated from a TFR using a ridge detector as:

1) Brevdo et al. method: This technique [19] aims at finding the best frequency curve $\Omega(t)$ in the TFR $S_{x}$, which maximizes the energy with a smoothness constraint through a total variation penalization term expressed as ( $c f$. ridge_detect_brvmask)

$$
\hat{\Omega}=\underset{\Omega}{\operatorname{argmax}} \int_{\mathbb{R}}\left|S_{x}(t, \Omega(t))\right|^{2} \mathrm{~d} t-\lambda \int_{\mathbb{R}}\left|\frac{\mathrm{d} \Omega}{\mathrm{d} t}(t)\right|^{2} \mathrm{~d} t,
$$

where $\lambda$ controls the importance of the smoothness constraint. For multi-component extraction (i.e. $I>1$ ), this method can be iterated after setting $S_{x}$ to zero in the vicinity of the previously detected ridge. 
2) Delaunay triangulation method: Frandrin proposes in [6] a new way to disentangle the different components in the time-frequency plane through a simplified representation provided by the Delaunay triangulation attached to the spectrogram zeros. For a particular Gaussian analysis window $g(t)=\pi^{-1 / 4} \mathbf{e}^{-\frac{t^{2}}{2}}$, the zeros $z_{n}=\omega_{n}+j t_{n}$ can be determined from the STFT, through Bargmann transform as:

$$
F_{x}^{g}(t, \omega)=\mathbf{e}^{-\frac{|z|^{2}}{4}} \mathcal{F}_{x}(z), \text { with } \mathcal{F}_{x}(z)=\int_{\mathbb{R}} A(z, \tau) x(\tau) d \tau,
$$

and $A(z, \tau)=\pi^{-1 / 4} \mathbf{e}^{-\frac{\tau^{2}}{2}-j \tau z+\frac{z^{2}}{4}}$.

It admits a Weierstrass-Hadamard factorization such as [6]:

$$
\mathcal{F}_{x}(z) \propto \prod_{n=1}^{\infty}\left(1-\frac{z}{z_{n}}\right) \mathbf{e}^{\frac{z}{z_{n}}+\frac{1}{2}\left(\frac{z}{z_{n}}\right)^{2}}
$$

Then, the Delaunay triangulation is performed over zeros $z_{n}$ and the domains attached to signal components are related to outlier edges ( $c f$. spz_delaunay_dom 4 a).

\section{DATA-DRIVEN METHODS}

The ASTRES toolbox also includes several implementations of the well known EMD method [7], which was reformulated and extended for two dimensional signals [13], [14]. Due to paper size limitation, we chose to focus on more recent developments allowing an unsupervised usage of SSA.

\section{A. Singular spectrum analysis}

SSA [8] can expand a signal in a sum of periodic components, trends and noise. It considers a finite length time series (e.g. a sampled signal) $s=\left\{s_{n}, n=1 \ldots N\right\}$ and a user parameter $L$. Its algorithm ( $c f$. s.sa) is described by:

1) Construction from $x$ of the trajectory Hankel matrix $X$ of size $L \times K$, with $K=N-L+1$.

2) Singular Value Decomposition (SVD) of $X$, to obtain

$$
X=\boldsymbol{U} \boldsymbol{\Sigma} \boldsymbol{V}^{T}=\sum_{i=1}^{R} X_{i}, \text { with } X_{i}=\sigma_{i} \boldsymbol{u}_{\boldsymbol{i}} \boldsymbol{v}_{\boldsymbol{i}}^{T},
$$

$\sigma_{i}$ being the singular values of $X$ and $\boldsymbol{u}_{\boldsymbol{i}}$ (resp. $\boldsymbol{v}_{\boldsymbol{i}}$ ) the column vectors of $\boldsymbol{U}$ (resp. $\boldsymbol{V}$ ).

3) Elementary components reconstruction from each $X_{i}$ by anti-diagonal averaging through

$$
x_{n}^{(i)}= \begin{cases}\frac{1}{n} \sum_{m=1}^{n}\left(X_{i}\right)_{m, n-m+1} & \text { for } 1 \leq n<L \\ \frac{1}{L} \sum_{m=1}^{L}\left(X_{i}\right)_{m, n-m+1} & \text { for } L \leq n \leq K \\ \frac{1}{N-n+1} \sum_{m=n-K+1}^{L}\left(X_{i}\right)_{m, n-m+1} & \text { for } K+1 \leq n \leq N .\end{cases}
$$

4) Unsupervised mode extraction through elementary components grouping, based on Hierarchical Clustering (HC) [9] ( $c f$. ssa_decomp). The main idea of this algorithm [20] is to look for the two nearest classes and to merge them to build a new class. This operation is iterated until the desired number of classes (i.e. I) is reached.
To compare two classes $C_{i}$ and $C_{j}$ containing several time series, the dissimilarity is defined as the minimal distance between two distinct time series of each class

$$
D\left(C_{i}, C_{j}\right)=\min _{x \in C_{i}, y \in C_{j}} d(x, y), d(x, y)=1-\frac{|<x, y>|}{\|x\|\|y\|},
$$

Thus, each extracted mode is obtained by summing the elementary components included into the resulting classes $C_{i}$.

\section{NUMERICAL SIMULATIONS}

\section{A. Time-frequency representations}

Fig. 2 compares the TFRs of the gravitational wave signal GW150914 [21], resulting from a binary black hole collision. These TFRs are computed using respectively, the Gabor transform (STFT using a Gaussian analysis window), the Morlet Wavelet transform and the S-transform, with their respective first- and second-order synchrosqueezed versions. The Reconstruction Quality Factor (RQF) given by $\operatorname{RQF}(x, \hat{x})=$ $10 \ln _{10}\left(\frac{\|x\|^{2}}{\|x-\hat{x}\|^{2}}\right)$ is indicated above each invertible TFR.

\section{B. Empirical mode decomposition}

For this experiment ( $c f$. Fig. 3), we consider a real-world multi-component audio signal recorded from a cello. This signal is analyzed using the synchrosqueezed STFT combined with the Brevdo et al. method described in Section III-D1, the EMD method and the proposed SSA method [9]. Synchrosqueezing leads to better separation results compared to other methods which are more likely to create interference.

\section{CONCLUSION AND FUTURE WORKS}

The ASTRES toolbox was introduced as a collection of Matlab functions for processing non-stationary and multicomponent signals. This toolbox which is freely available online, unifies into the same framework several recent techniques developed into the ASTRES project. Some capabilities of these methods designed for efficient TFRs computation and mode extraction, were also illustrated by application examples on real world signals. Future works consist in theoretically strengthening these tools, and in proposing new applications.

\section{REFERENCES}

[1] P. Flandrin, Time-Frequency/Time-Scale analysis. Acad. Press, 1998.

[2] F. Auger and P. Flandrin, "Improving the readability of time-frequency and time-scale representations by the reassignment method," IEEE Trans. Signal Process., vol. 43, no. 5, pp. 1068-1089, May 1995.

[3] I. Daubechies, J. Lu, and H.-T. Wu, "Synchrosqueezed wavelet transforms: An empirical mode decomposition-like tool," Applied and Computational Harmonic Analysis, vol. 30, no. 2, pp. 243-261, 2011.

[4] F. Auger, P. Flandrin, Y. Lin, S. McLaughlin, S. Meignen, T. Oberlin, and $\mathrm{H}$. Wu, "TF reassignment and synchrosqueezing: An overview," IEEE Signal Process. Mag., vol. 30, no. 6, pp. 32-41, Nov. 2013.

[5] S. Meignen, T. Oberlin, and S. McLaughlin, "A new algorithm for multicomponent signals analysis based on synchrosqueezing: with an application to signal sampling and denoising," IEEE Trans. Signal Process., vol. 60, no. 11, pp. 5787-5798, Nov. 2012.

[6] P. Flandrin, "Time-frequency filtering based on spectrogram zeros," IEEE Signal Process. Lett., vol. 22, no. 11, pp. 2137-2141, 2015.

[7] N. E. Huang, Z. Shen, S. R. Long, M. C. Wu, H. H. Shih, Q. Zheng, N.C. Yen, C. C. Tung, and H. H. Liu, "The empirical mode decomposition and the Hilbert spectrum for nonlinear and non-stationary time series analysis," Proc. of the Royal Society of London A: Math., Physical and Engineering Sciences, vol. 454, no. 1971, pp. 903-995, 1998. 

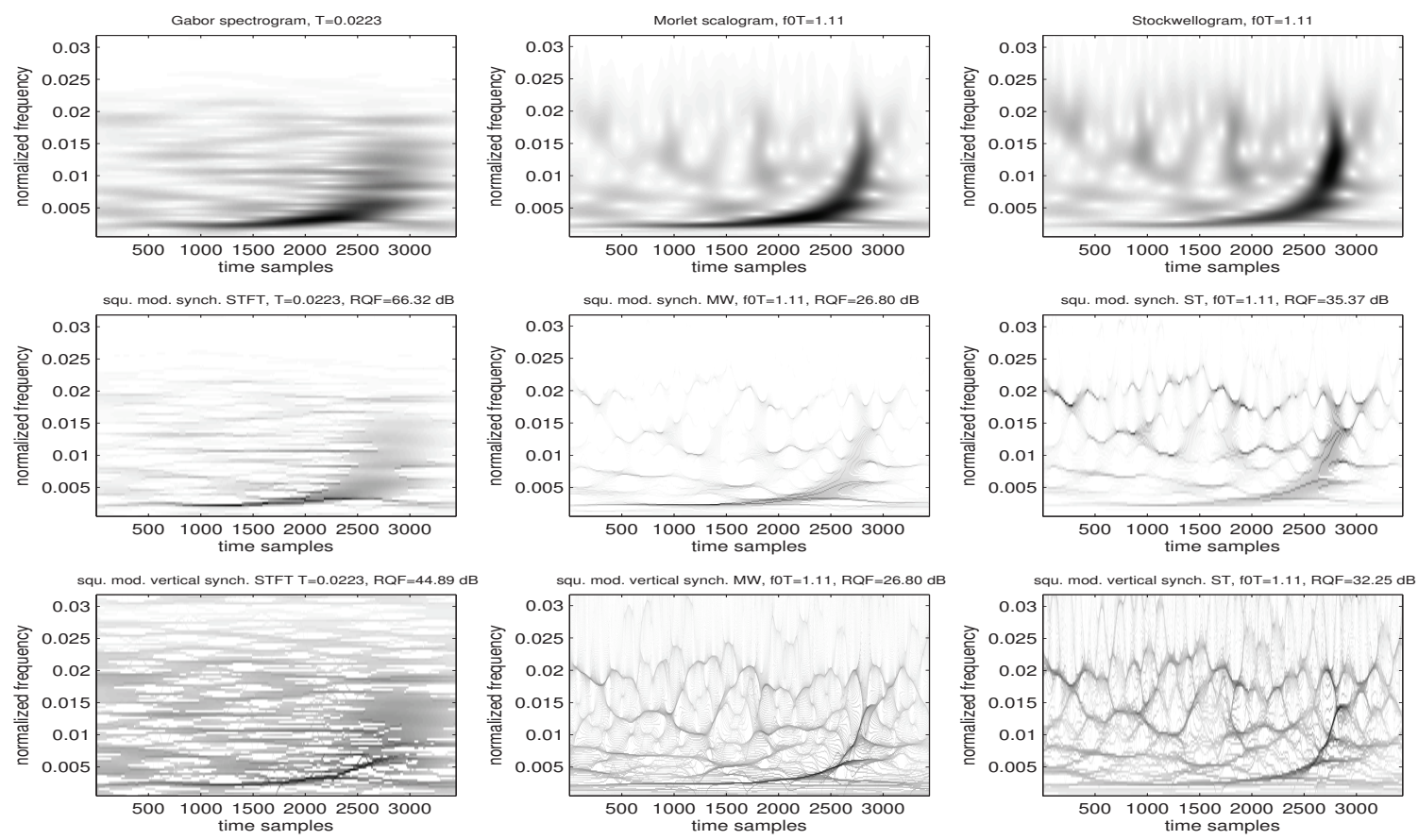

Fig. 2. Comparison between the Gabor STFT, Morlet CWT and the Stockell transform applied on the Livingston GW150914 signal [21].
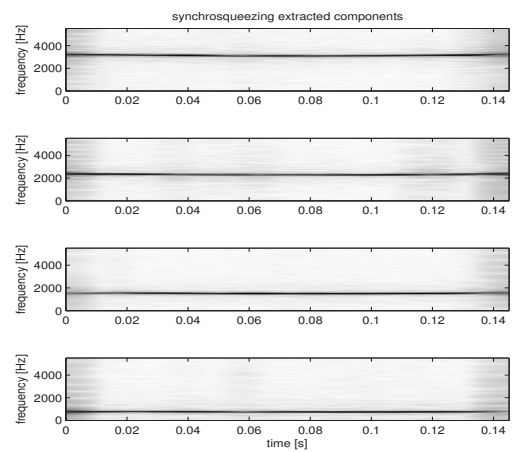

(a) synchrosqueezed STFT
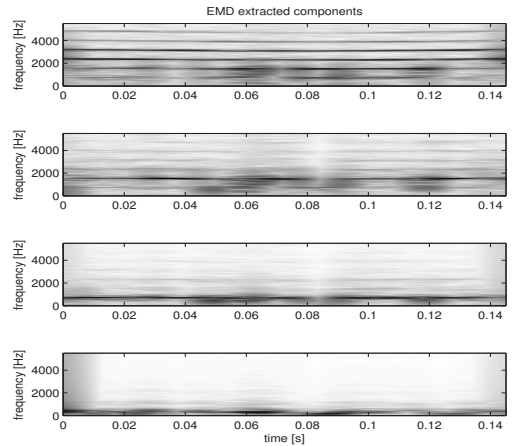

(b) EMD
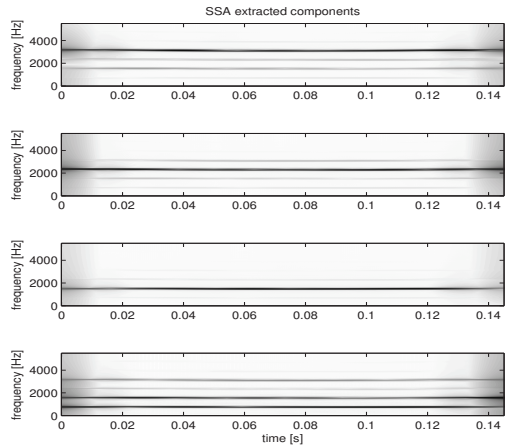

(c) SSA

Fig. 3. Comparison of the extracted modes resulting from a real-world audio signal, using synchrosqueezed STFT, EMD and the proposed SSA method.

[8] J. Elsner and A. Tsonis, Singular Spectrum Analysis, A New Tool in Time Series Analysis. Plenum Press, 1996.

[9] J. Harmouche, D. Fourer, F. Auger, P. Flandrin, and P. Borgnat, "One or two components? the singular spectrum analysis answers," in Structured Low-Rank Approximation (SLRA'2015), Grenoble, France, Jun. 2015.

[10] D. Fourer, F. Auger, and P. Flandrin, "Recursive versions of the Levenberg-Marquardt reassigned spectrogram and of the synchrosqueezed STFT," in Proc. IEEE ICASSP, Mar. 2016, pp. 4880-4884.

[11] T. Oberlin, S. Meignen, and V. Perrier, "Second-order synchrosqueezing transform or invertible reassignment? Towards ideal time-frequency representations," IEEE Trans. Signal Process., vol. 63, no. 5, pp. 13351344, Mar. 2015.

[12] D. Fourer, F. Auger, K. Czarnecki, S. Meignen, and P. Flandrin, "Chirp rate and instantaneous frequency estimation: application to recursive vertical synchrosqueezing," IEEE Signal Process. Lett., 2017.

[13] T. Oberlin, S. Meignen, and V. Perrier, "An alternative formulation for the empirical mode decomposition," IEEE Trans. Signal Process., vol. 60, no. 5, pp. 2236-2246, 2012.

[14] J. Schmitt, N. Pustelnik, P. Borgnat, P. Flandrin, and L. Condat, "2D prony-huang transform: A new tool for 2D spectral analysis," IEEE
Trans. Image Process., vol. 23, no. 12, pp. 5233-5248, 2014.

[15] R. Stockwell, L. Mansinha, and R. Lowe, "Localization of the complex spectrum: the S-transform," IEEE Trans. Signal Process., vol. 44, no. 4, pp. 998-1001, Apr. 1996.

[16] D. Fourer, F. Auger, and J. Hu, "Reassigning and synchrosqueezing the Stockwell transform: Complementary proofs," Tech. Rep., Dec. 2015. [Online]. Available: http://hal.archives-ouvertes.fr/hal-01467244

[17] F. Auger, E. Chassande-Mottin, and P. Flandrin, "Making reassignment adjustable: the Levenberg-Marquardt approach," in Proc. IEEE ICASSP, Mar. 2012, pp. 3889-3892.

[18] T. Oberlin and S. Meignen, "The second-order wavelet synchrosqueezing transform," in Proc. IEEE ICASSP, Mar. 2017, p. (to appear).

[19] E. Brevdo, N. S. Fuckar, G. Thakur, and H.-T. Wu, "The synchrosqueezing algorithm: a robust analysis tool for signals with time-varying spectrum," Arxiv preprint, 2011.

[20] J. H. Ward, "Hierarchical grouping to optimize an objective function," JASA, vol. 58, pp. 236-244, 1963.

[21] P. Flandrin, "A note on the time-frequency analysis of GW150914," École normale supérieure de Lyon, Tech. Rep., Sep. 2016. [Online]. Available: https://hal-ens-lyon.archives-ouvertes.fr/ensl-01370441 